\title{
La descripción matemática de la detección del fármaco Danazol, mediante un proceso electroanalítico con uso del nitruro de carbono.
}

\author{
Volodymyr V. Tkach ${ }^{1,2}$, Marta V. Kushnir ${ }^{1}$, Sílvio C. de Oliveira ${ }^{2}$, Yana G. Ivanushko ${ }^{3}$, Nataliia A. \\ Stratiychuk ${ }^{1}$, Oleksandra V. Ahafonova ${ }^{3}$, Petro I. Yagodynets' ${ }^{\prime 1}$ \\ 1 Universidad Nacional de Chernivtsi, 58000, Calle de Kotsyubyns'ky, 2, Chernivtsi, Ucrania \\ 2Universidade Federal de Mato Grosso do Sul, Instituto de Química, 79074-460, Av. Sen. Felinto Muller, 1555, Unidade XI, Campo \\ Grande, MS, Brasil \\ 3Universidad Estatal de Medicina de Bucovina, 58000, Pl. Teatral, 9, Chernivtsi, Ucrania
}

Recibido el 07 de Febrero del 2019, Aceptado el 05 de Mayo del 2019.

\section{Resumen}

Mediante un análisis teórico ha sido prevista la posibilidad de realizar el proceso de la determinación electroanalítica de Danazol sobre el cátodo, modificado por el nitruro de carbono. El modelo matemático, relativo al proceso electroanalítico, ha sido desarrollado y analizado mediante la teoría de estabilidad lineal y de análisis de bifurcaciones. Ha sido comprobada teóricamente la eficiencia del nitruro de carbono como modificador de cátodo para la electroreducción de Danazol. Otrosí, la zona de la dependencia lineal entre la concentración del fármaco y el parámetro electroquímico se hace bastante ancha para el uso en detección en formas farmacéuticas y líquidos biológicos. Por otro lado, el comportamiento oscilatorio, bien como el monotónico, también se puede realizar.

Palabras clave: prevención de fraude deportiva, Danazol, nitruro de carbono, sensores electroquímicos, estado estacionario estable.

\section{Introducción}

Danazol [1-6] es un fármaco, usado para tratar endometriosi s, dolor antes y durante los períodos menstruales, dolor durante y después de la acti vi dad sexual y sargrado intenso o i rregular El Danazol también se usa para tratar la enfermedad de senos fi broquísti cos (senos i nflamados, sensibles con bultos no cancerosos) cuando a otros tratami entos falta rendi mi ento. Otrosí, se usa para prevenir ataques en las personas con angi edema hereditario. El Danazol pertenece a una clase de medi camentos llamados hormonas androgéni cas.

A pesar de sus efectos positivos, el uso de Danazol suele acompañarse por efectos secundari os [7-12] como:

- Acné

- Di smi nuci ón en el tamaño de los senos

- Aumento de peso

- Pi el o cabello grasoso

- Rubor

- Sudoración

- Sequedad vaginal, ardor, pi cazón o sangrado

- Nervi osi smo

- Irri tabi li dad

- Ausenci a del ci clo menstrual, manchado o cambi os en el ci clo menstrual.
Por cuenta de su acción esti mulante, Danazol es considerado un dopante bioquímico $\mathrm{y}$, por consi gui ente, prohi bi do para uso ercompeti ci ones deportivas [13-14]. Por eso, el desarrollo de métodos de la cuanti fi cación de su concentaci ón es realmente actual [15-17], y el uso de métodos electroanalíti cos podría ser una buena soluci ón para esta tarea

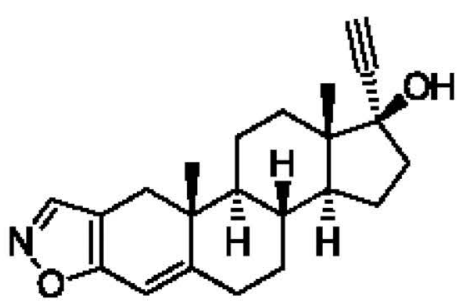

Fig. 1 - Danazol

La determi nación electroanalíti ca del Ianazol ya ha si do realizada en los trabajos [18-19]. Naturalmente, la determi nación del fármaco se reali zó por vía catódi ca, si endo el anali to reduci do mayoritariamente por la ruptra del el anillo i soxazóli co. Un material interesante con potencial para ser modi fi cador de cátodo para la educción electroanalítica del Danazol podría ser el 
oxi hi dróxi do de vanadi o $(\mathrm{VO}(\mathrm{OH})$ un material análogo al oxi hi dróxi dode cobalto, estable en la banda de $\mathrm{pH}$ entre 3 y 14 , pero con un comportami ento electroquímico más i ncli nado para catódi co,pudi endo ser sustanci a acti va o medi ador.

Por otro lado, por ahora, ni ngún método electroanalítico, que use el oxihidróxido de vanadi o con Danazol o cualqui er otro anali to, ha si do desarrollado. Solamente un trabajo [20], en que $\mathrm{VO}(\mathrm{OH})$ es usado como modificador de electrodo en condensadores, ha si do publi cado. $\mathrm{B}$ desarrollo y uso práctico de nuevos métodos electroanalíticos puede impicar la resolución de ci ertos problemas, como:

-La necesidad de combinar los parámetros de estabi li dad de sustanci as, que partici pan en el acto electroanalítico, con los de la mayor efi ci enca del proceso electroanalíti co

-La posi bi lidad de la apari ciórde inestabi li dades electroquímicas, que ya han sido observadas durante el desempeño del oxi hi dróxi do de cobalto [21-22], que es un compuesto semejante.

Habi endo en vi sta loci tado previ amentẹ el análi si s teórico a priori es necesario para describir un sistema con potencial electroquímico. Así si endo, el objeti vo general de este trabajo se desti na a este análi sis, apli cado para el si stema conla detección electroquímica del Danazol en el cátodo, modi fi cado por $\mathrm{VO}(\mathrm{OH})$. El modelo matemático, capaz de describir adecuadamente el comportami ento del si stema, se desarrolla y anali za medi ante la teoría de estabi li dad li neal y análi si s de bi furcaciones. Del análisis se hacen las conclusi ones acerca de la efi ci enci a del proceso en la detecci ón electroanalíti ca del Danazol y, tambi én se hace comparar el comportamiento de este proceso con él de los semejantes [23-25].

\section{El sistema y su modelo}

Como siendo un derivado isoxazólico, en la primera etapa el Danazol se reducirá hasta la apertura del anillo, conforme la Fig. 2

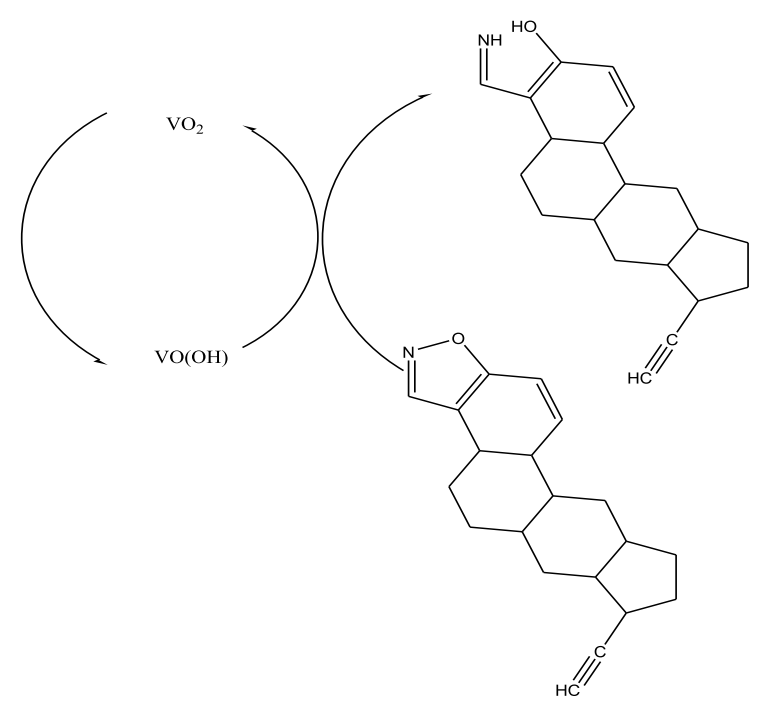

Fig. 2 - La primera etapa de la reducción electroquímica de Danazol

En la segunda etapa se realiza la reducción parcial del enlace triplo, formando el duplo, conforme la Fig. 3

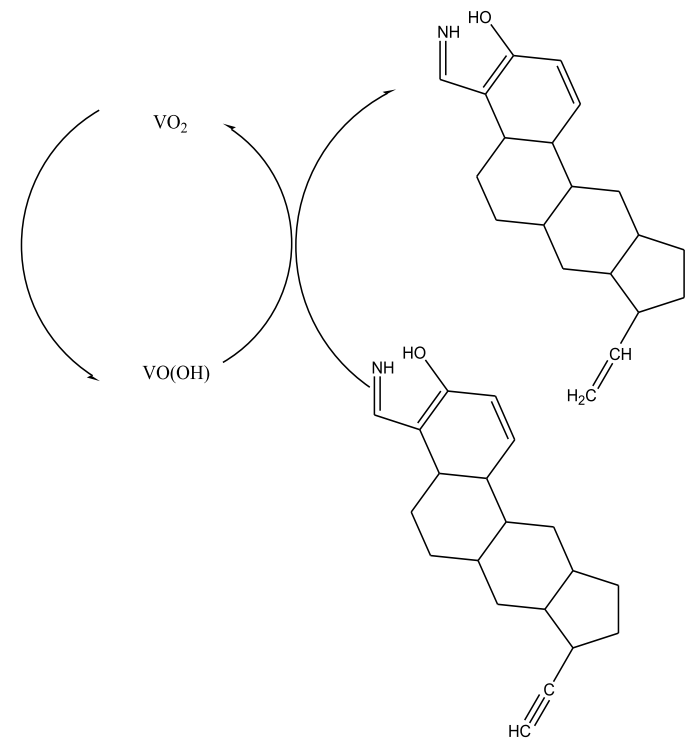

Fig. 3 - La segunda etapa de la reducción electroquímica de Danazol

La reducción del grupo imina también puede ser realizada según la Fig. 4 


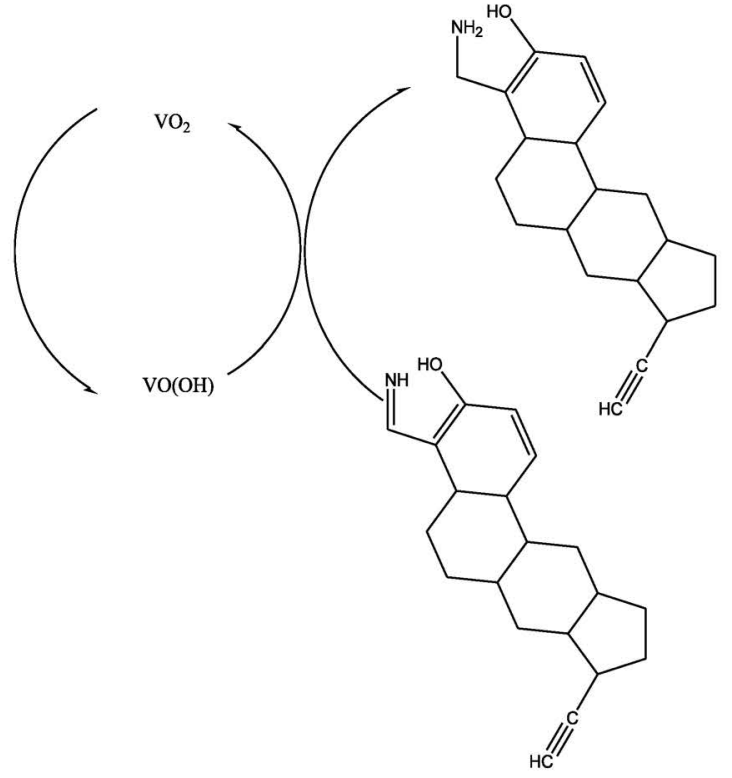

Fig. 4 - La segunda etapa de la reducción electroquímica de Danazol (escenario 2)

Así, para describir el comportamiento electroanalítico del sistema con la detección electroanalítica del Danazol con el oxihidróxido de vanadio, introducimos las tres variables:

c - la concentración del Danazol en la capa presuperficial

$\mathrm{p}$ - la concentración del producto de la ruptura del anillo heterocíclico

$\theta$ - el grado de recubrimiento de la superficie de cátodo por el oxihidróxido de vanadio.

Para simplificar el modelo, suponemos que el reactor se agite intensamente, lo que nos deja menospreciar el flujo de convexión. Otrosí, suponemos que el electrólito de soporte esté presente en su exceso, dejándonos menospreciar el flujo de migración. Suponemos, también, que el perfil concentracional de las sustancias en la capa pre-superficial sea lineal, y su espesor, constante e igual a $\delta$.

Es posible mostrar que, considerando lo citado, el comportamiento del sistema será descrito por un conjunto de ecuaciones diferenciales conforme:

$$
\left\{\begin{array}{c}
\frac{d c}{d t}=\frac{2}{\delta}\left(\frac{\Delta}{\delta}\left(c_{0}-c\right)-r_{2}\right) \\
\frac{d p}{d t}=\frac{2}{\delta}\left(r_{2}-r_{31}-r_{32}\right) \\
\frac{d \theta}{d t}=\frac{1}{G}\left(r_{2}+r_{31}+r_{32}-r_{3}\right)
\end{array}\right.
$$

En que D es el coeficiente de difusión del danazol, c0 su concentración en la capa pre-superficial, $G$ es la concentración superficial máxima del oxihidróxido de vanadio en la superficie del cátodo y los parámetros $r$ son las velocidades de las reacciones de las respectivas reacciones, que se pueden calcular conforme:

$$
\begin{aligned}
& r_{2}=k_{2} c(1-\theta)^{2} \\
& r_{31}=k_{31} p(1-\theta)^{2} \\
& r_{32}=k_{32} p(1-\theta)^{2} \\
& r_{3}=k_{3} \theta \exp \left(-\frac{F \varphi_{0}}{R T}\right)
\end{aligned}
$$

En que los parámetros $\mathrm{k}$ son constantes de velocidades de las respectivas reacciones, $\mathrm{F}$ es el número de Faraday, $\varphi 0$ es el salto de potencial en la doble capa eléctrica (DCE), relacionado al potencial de carga cero, $\mathrm{R}$ es la constante universal de gases y $\mathrm{T}$ es la temperatura absoluta.

Se trata, de hecho, de un sistema parecido con él de detección del ácido iboténico en las mismas condiciones [25]. Sin embargo, como las propiedades básicas del anillo isoxasólico no se manifiestan en este sistema, no habrá influencias adicionales de las etapas químicas en la DCE, lo que hará el estado estacionario más estable, y el sistema electroanalítico aún más eficiente, como se muestra abajo.

\section{Resultados y discusión}

Para investigar el comportamiento del sistema con la detección electroquímica de Danazol, realizada mediante un proceso de reducción, asistida por el oxihidróxido de vanadio, analizamos el conjunto de ecuaciones diferenciales (1), mediante la teoría de estabilidad linear. Los elementos estacionarios de la matriz funcional de Jacobi se presentan conforme:

$$
\left(\begin{array}{lll}
a_{11} & a_{12} & a_{13} \\
a_{21} & a_{22} & a_{23} \\
a_{31} & a_{32} & a_{33}
\end{array}\right)
$$

Siendo:

$$
\begin{aligned}
& a_{11}=\frac{2}{\delta}\left(-\frac{\Delta}{\delta}-k_{2}(1-\theta)^{2}\right) \\
& a_{12}=0
\end{aligned}
$$




$$
a_{13}=\frac{2}{\delta}\left(2 k_{2} c(1-\theta)\right)
$$

$$
a_{21}=\frac{2}{\delta}\left(k_{2}(1-\theta)^{2}\right)
$$

$$
a_{22}=\frac{2}{\delta}\left(-k_{31}(1-\theta)^{2}-k_{32}(1-\theta)^{2}\right)
$$

$$
\begin{aligned}
& a_{23}=\frac{2}{\delta}\left(-2 k_{2} c(1-\theta) \quad \exp (-\alpha c)+\right. \\
& \left.2 k_{31} p(1-\theta)+2 k_{32} p(1-\theta)\right)
\end{aligned}
$$

$$
a_{31}=\frac{1}{G}\left(k_{2}(1-\theta)^{2}\right)
$$

$$
a_{32}=\frac{1}{G}\left(k_{31}(1-\theta)^{2}+k_{32}(1-\theta)^{2}\right)
$$

$$
\begin{aligned}
& a_{33}=\frac{1}{G}\left(-2 k_{2} c(1-\theta)-2 k_{31} p(1-\right. \\
& \theta) \quad- \\
& 2 k_{32} p(1-\theta) \quad-k_{3} \exp \left(-\frac{F \varphi_{0}}{R T}\right)+ \\
& \left.\xi k_{3} \theta \exp \left(-\frac{F \varphi_{0}}{R T}\right)\right)
\end{aligned}
$$

Observando los elementos de la diagonal principal (6), (10) y (14), se puede averiguar que el comportamiento oscilatorio se puede realizar en este sistema. Sin embargo, al contrario del caso del ácido iboténico [25], su probabilidad se reducirá, pudiendo la estabilidad realizarse apenas por causa de las influencias de la etapa electroquímica en la DCE. Las oscilaciones se esperan menos frecuentes, pero de mayor amplitud que en [25].

Para investigar la estabilidad de estado estacionario, aplicamos al conjunto de ecuaciones diferenciales (1) el criterio Routh-Hurwitz. Evitando las expresiones grandes, introducimos nuevas variables, para que el determinante del Jacobiano se describa como:

$$
\frac{4}{\delta^{2} G}\left|\begin{array}{ccc}
-\kappa-\Xi & 0 & \Omega \\
\Xi & -X & -\Omega+\Lambda \\
\Xi & X & -\Omega-\Lambda-P
\end{array}\right|
$$

Abriendo los paréntesis y aplicando el requisito Det $\mathrm{J}<0$, saliente del criterio, se obtiene la condición de estabilidad del estado estacionario, descrita como:

$$
-2 \kappa X \Omega-(\kappa+\Xi) X P<0
$$

Representando un proceso electroanalítiico eficiente, controlado por la difusión de analito. Contrariamente al caso, descrito en [25], la zona topológica de estabilidad de estado estacionario abarca una mayor variedad de valores de parámetros, $\mathrm{y}$, así, la dependencia lineal entre la concentración de danazol y el parámetro electroquímica se mantendrá en la medida más ancha de concentraciones del analito.

La condición de inestabilidad monotónica, correspondiente al límite de detección, se describe como:

$$
-2 \kappa X \Omega-(\kappa+\Xi) X P=0
$$

En este punto coexisten varios estados estacionarios, todos inestables. El sistema escoge apenas uno, y este se autodestruye, cuando las condiciones del sistema se alteran.

Se puede describir el comportamiento de este sistema por un conjunto de dos ecuaciones diferenciales. Suponiendo el comportamiento permanentemente estacionario del producto del proceso en la Fig. 2, se puede "unir" las reacciones, descritas en las figuras 2 y 3 (escenario 1) y 2 y 4 (escenario 2), removiendo la variable $\mathrm{p}$ del conjunto de ecuaciones. Así, el sistema dinámico se simplifica, transformándose en bivariante.

\section{Conclusiones}

El análisis teórico de la posibilidad de la detección electroquímica del Danazol, asistida por el oxihidróxido de vanadio trivalente, dejó concluir que:

-Se trata de un sistema electroanalítico eficiente, controlado por la difusión, más eficaz que en el caso de ácido iboténico

-La dependencia lineal entre la concentración de Danazol y el parámetro electroquímico se realiza en la amplia zona de concentraciones

-El comportamiento oscilatorio en este sistema solo se realiza por causa de factores de influencia de la etapa electroanalítica en la doble capa.

\section{Referencias}

1. http://www.medicinescomplete.com/mc/martindale/c urrent/9025-v.htm, acceso a 29 de enero de 2019

2. Th. Lemke, .D. Williams, Foye's Principals of Medicinal Chemistry, 2010, Nueva York, Lipincott Williams \& Wilkins

3. W. Dmowski, H. Scholer, V. Mahesh, R. Greenblatt, Fertil Steril., 22(1971), 9 
4. S. Luisi, S. Razzi, L. Lazzeri et al., Fertil. Steril., 92(2009), 1251

5. https://www.wada-ama.org/en/content/what-isprohibited/prohibited-at-all-times/anabolic-agents, acceso a 29 de enero de 19

6. A.M. Marzouk, G. Maatog., Z. Naturforsch, 69(2014), 245

7. V. Selak, C. Farguhar, A. Prentice, A. Singla, Colchrane Datab. Syst. Rev., 4(2001), 68

8. H. Farkas, I. Czaller, D. Csuka et al., J. Clin. Pharm., 66(2009), 419

9. Y. Nagata, G. Nakamura, M. Kusuda, J. Obst. Gyn. Res., 8(1962), 229

10. W. Li, X. Gu, R. Fu et al., Clin. Appl. Thromb., 22(2016), 727

11. G. Szeplaki, L. Varga, Sz. Valentin et al., J. Allerg. Clin. Immun., 115(2005), 864

12. https://www.ejmanager.com/mnstemps/89/891385020637.pdf?t=1548795804, acceso a 29 de enero de 2019

13. R. Kumar, Br. J. Sport. Med., 44(2010), 1

14.

http://pilarmartinescudero.es/dic13/automatizacion muestras_analisis_sustdopantes.pdf acceso a 29 de enero de 2019

15. R. Shanmugam, A. Kirthi, K. Madhuri et al., Hygeia J. D. Med., 8(2016), 27

16. Y. Ye, J. Lin, X. Zhang et al., Chin. J. Pharm. Anal., $28(2008), 216$

17. A. Kirthi, R. Shanmugam, S. Lakshmi et al., Asian J. Pharm. Anal., 6(2016), 227

18. M. Al-Omar, A. Al-Majed, M. Sultan et al., J. Pharm. Biomed. Anal., 37(2005), 199

19. A. Alghamdi, F. Belal, M. Al-Omar, J. Pharm. Biomed. Anal., 41(2006), 989

20. Y. Zhang, X. Jing, Mat. Lett., 205(2017), 1

21. Stadnik O. Synthesis, Electrochemical and Photoelectrochemical Properties of the Oxidehydroxide Compounds of Cobalt, Diss. Kand. Chim. N. - Kyiv. -2011

22. O. Stadnik, N. Ivanova, Y. Boldyrev, 218th Int. Electrochem. Soc. Meeting. Abstract \# 2240, http://ma.ecsdl.org/content/MA201002/38/2240.full. pdf Accessed at 8th of August 2015

23. V.V. Tkach, I. L. Kukovska, Ya. G. Ivanushko et al., El-Cezeri J. Sci. Eng., 5(2018), 292

24. V.V. Tkach, S.C. de Oliveira, S. Lukanova et al., Appl. J. Env. Eng. Sci., 4(2018), 33
25. V.V. Tkach, N.M. Storoshchuk, M.V. Kushnir et al., Act. Probl. Biol. Ecol. Chem., 15(2018), 127, en ucraniano 